\title{
Editorial of cross-layer design issues, challenges and opportunities for future intelligent heterogeneous networks
}

\author{
Muhammad Khalil Afzal ${ }^{1}$ - Wazir Zada Khan ${ }^{2} \cdot$ Tariq Umer $^{1} \cdot$ Byung-Seo Kim ${ }^{3}$. Shui Yu ${ }^{4}$
}

Published online: 10 August 2018

○) Springer-Verlag GmbH Germany, part of Springer Nature 2018

The concept and operations of data communications are based on the integration of various heterogeneous wireless networks such as mobile communication networks $(3 \mathrm{G} / 4 \mathrm{G} /$ LTE/5G), wireless sensor networks (WSNs), wireless local area networks (WLANs), wireless mesh networks (WMNs), wireless body area networks (WBANs), etc.. These wireless networks are providing backbone for future generation communications technologies. Unique characteristics of wireless networks such as mobility, high error rates, and multipath propagations bring the new challenges to traditional transmission control protocol/internet protocol (TCP/IP)-based protocol stack which was developed mainly for wired networks. The unexpected packet losses in wireless networks significantly degrade the networks' performances. On the other hand, quality of service (QoS) is another challenge for the wireless networks which enable users to share multimedia data while moving in real time.

For the efficient and controlled communications in the future generation networks, it is very important to design and develop smart and state-of-the-art algorithms. Even

\author{
Muhammad Khalil Afzal \\ khalilafzal@ciitwah.edu.pk \\ Wazir Zada Khan \\ wazirzadakhan@jazanu.edu.sa \\ Tariq Umer \\ t_umer@yahoo.com \\ Byung-Seo Kim \\ jsnbs@hongik.ac.kr \\ Shui Yu \\ shui.yu@deakin.edu.au
}

1 Department of Computer Science, COMSATS University Islamabad, Wah Campus, Islamabad, Pakistan

2 Jazan University, Jizan, Kingdom of Saudi Arabia

3 Department of Software and Communications Engineering, Hongik University, Sejong, South Korea

4 School of Information Technology, Deakin University, Geelong, Australia though the protocol architecture traditionally follows strict layering principle, the lack of coordination among the layers rather limits the performances of wireless networks. In the future wireless networks, different network parameters like energy efficiency, mobility management, security, cooperation, and QoS require optimization which can be achieved through cross-layer design. The core idea of the cross- layer design is not only to maintain the functionalities associated with the conventional original layers, but also to allow coordination, integration, and joint optimization of protocols. The goal of this special issue is to solicit the state-of-theart and novel protocols, and methodologies that embark on cross-layer design issues and opportunities for future intelligent heterogeneous wireless networks. In this special issue, we received seven submissions in total and after rigorous reviews, three papers were accepted. These papers cover a broad range of topics related to cross-layer designs.

In mobile peer-to-peer (MP2P) systems, flooding introduces a large amount of network traffic and increases the communications redundancy. To deal with these issues, in the first contribution "PCSM: An Efficient Multihop Proximity aware Clustering Scheme for Mobile Peer-to-Peer Systems" by Moufida Rahmani et al., introduces a multi-hop clustering-based scheme, called proximity aware clustering scheme for mobile peer-to-peer systems (PCSM). In PCSM, the mismatch between P2P overlays and network layer is reduced by considering the physical proximity between the peers. In the propose scheme, the process of joining the networks and connecting a new peer to a cluster head is based on three elements, the number of physical hops, cluster size, and the availability of cluster head. The results show that as compared to existing cluster based P2P overlays schemes, the PCSM shows improvements in load balancing and routing overhead. PCSM also improves the searching process by reducing the average file discovery delay and false negative ratio.

Battery power is considered among the most precious resource for underwater WSNs (UWSNs). Lifespan of a 
UWSN node is directly associated with it battery power consumption. The communications over long distances and redundant data transmissions along with other generic challenges faced by UWSN result in fast battery dissipation which leads to void hole creation. To prevent void creation in advance with reduced redundant transmission, the article, titled "Fair energy management with void hole avoidance in intelligent heterogeneous underwater WSNs" by Nadeem Javaid et al., proposes a routing protocol, called a novel routing strategy for void hole detection and avoidance. For transmitting data packets, a set of forwarder nodes are selected based on node depth and energy, and for reducing duplicated transmissions, the forwarding communication range is logically divided into sub-forwarding areas. The obtained results show that in terms of packet delivery ratio, number of duplicated packets, an end-to-end delay and total energy tax the proposed scheme outperformed the existing schemes.

Energy waste at physical and medium access control (MAC) layers due to erroneous bits in frame and collisions among frames cause dropping frames in WSNs. As stated earlier, battery power is considered among the most precious resources in WSNs. Successful frame transmissions and reducing energy waste due to packet drop can be handled through a combined cross-layer model. Sankalita Biswas et al. in their article "Cross-layer Energy Model for
Beacon-enabled 802.15.4 Networks" propose a cross-layer energy model for communication in beacon-enabled IEEE 802.15.4 WSNs. In the proposed model, two dimensional Markov models are employed for back-off and clear channel assessment (CCA) along with a cross-layer energy model. This work aims to reduce the cross-layer error rate using different MAC and physical parameters for computing per bit energy consumption of an entire frame transmission cycle. Results show that the longer frame lengths (within the range specified by the IEEE standard) reduce energy consumptions. It is also observed that transmissions without acknowledgement reception can also help in energy saving.

This special issue is the outcome from the joint hard work of the authors, reviewers, and the editorial team. We would like to thank all the people involved in this special issue. We also appreciate the strong support from Dr. Vincenzo Loia, the Editor-in-chief of Journal of Ambient Intelligence and Humanized Computing.

Publisher's Note Springer Nature remains neutral with regard to jurisdictional claims in published maps and institutional affiliations. 